OPEN ACCESS

Edited by:

Kui Li,

Institute of Animal Sciences (CAAS),

China

Reviewed by:

Amanda K. Lindholm-Perry, U.S. Meat Animal Research Center

(USDA-ARS), United States

Kerong Shi,

Shandong Agricultural University,

China

*Correspondence:

Francisco Peñagaricano fpenagarican@wisc.edu

Specialty section:

This article was submitted to

Livestock Genomics,

a section of the journal

Frontiers in Genetics

Received: 27 October 2021 Accepted: 15 December 2021

Published: 04 January 2022

Citation:

Novo LC, Cavani L, Pinedo $P$, Melendez $P$ and Peñagaricano $F$ (2022) Genomic Analysis of Visceral Fat Accumulation in Holstein Cows.

Front. Genet. 12:803216.

doi: 10.3389/fgene.2021.803216

\section{Genomic Analysis of Visceral Fat Accumulation in Holstein Cows}

\author{
Larissa C. Novo ${ }^{1}$, Ligia Cavani ${ }^{1}$, Pablo Pinedo ${ }^{2}$, Pedro Melendez ${ }^{3}$ and \\ Francisco Peñagaricano ${ }^{1 *}$
}

${ }^{1}$ Department of Animal and Dairy Sciences, University of Wisconsin, Madison, WI, United States, ${ }^{2}$ Department of Animal Sciences, Colorado State University, Fort Collins, CO, United States, ${ }^{3}$ School of Veterinary Medicine, Texas Tech University, Amarillo, TX, United States

Visceral fat is related to important metabolic processes, including insulin sensitivity and lipid mobilization. The goal of this study was to identify individual genes, pathways, and molecular processes implicated in visceral fat deposition in dairy cows. Data from 172 genotyped Holstein cows classified at slaughterhouse as having low $(n=77$; omental fold $<5 \mathrm{~mm}$ in thickness and minimum fat deposition in omentum) or high ( $n=95$; omental fold $\geq 20 \mathrm{~mm}$ in thickness and marked fat deposition in omentum) omental fat were analyzed. The identification of regions with significant additive and non-additive genetic effects was performed using a two-step mixed model-based approach. Genomic scans were followed by gene-set analyses in order to reveal the genetic mechanisms controlling abdominal obesity. The association mapping revealed four regions located on BTA19, BTA20 and BTA24 with significant additive effects. These regions harbor genes, such as SMAD7, ANKRD55, and the HOXB family, that are implicated in lipolysis and insulin tolerance. Three regions located on BTA1, BTA13, and BTA24 showed marked non-additive effects. These regions harbor genes MRAP, MIS18A, PRNP and TSHZ1, that are directly implicated in adipocyte differentiation, lipid metabolism, and insulin sensitivity. The gene-set analysis revealed functional terms related to cell arrangement, cell metabolism, cell proliferation, cell signaling, immune response, lipid metabolism, and membrane permeability, among other functions. We further evaluated the genetic link between visceral fat and two metabolic disorders, ketosis, and displaced abomasum. For this, we analyzed 28k records of incidence of metabolic disorders from 14k cows across lactations using a single-step genomic BLUP approach. Notably, the region on BTA20 significantly associated with visceral fat deposition was also associated with the incidence of displaced abomasum. Overall, our findings suggest that visceral fat deposition in dairy cows is controlled by both additive and non-additive effects. We detected at least one region with marked pleiotropic effects affecting both visceral fat accumulation and displaced abomasum.

Keywords: abdominal fat, genomic scan, fat deposition, metabolic disorders, displaced abomasum

\section{INTRODUCTION}

Fat deposition occurs essentially in three regions, namely intramuscular, subcutaneous, and around visceral organs. These fat depots defer in structural organization, cellular size, biological function, and immunological and metabolic characteristics. Historically, visceral fat was considered to protect and insulate the internal organs; however, its critical role as a form of energy storage and 
endocrinological signaling has been recently recognized (Booth et al., 2016). Indeed, visceral adipose tissue carries out relevant functions, including lipogenesis to store surplus energy as triglycerides during periods of overnutrition, lipolysis to release energy as free fatty acids during periods of undernutrition, and secretion of a broad spectrum of active molecules, such as proinflammatory cytokines (Item and Konrad, 2012). Moreover, visceral adiposity has been linked to several metabolic disorders, including impaired glucose and lipid metabolism, and insulin resistance (Konrad and Wueest, 2014).

Dairy cows typically experience a state of negative energy balance around parturition and early lactation, when the energy demand for maintenance and milk production exceeds that of dietary energy intake (Pascottini et al., 2020). This negative energy balance leads to fat mobilization, and consequently, an increase in plasma concentrations of non-esterified fatty acids (NEFA), which are used as a fuel source by peripheral tissues and the mammary gland for milk fat synthesis. Visceral adipocytes in dairy cows are more metabolically active and sensitive to lipolysis than subcutaneous adipocytes, and hence, visceral adipose tissue is a key player in metabolic health during the transition period (Ji et al., 2014b). Note that extreme lipid mobilization is associated with different metabolic disorders, including displacement of the abomasum, ketosis, and fatty liver (Hostens et al., 2012; Contreras et al., 2015). Displacement of the abomasum, a condition in which the abomasum becomes enlarged with fluid and gas with subsequent migration to the left or right within the abdominal cavity, results in reduced rumination, decreased milk production, increased veterinary costs, and premature culling.

There is growing evidence that body fat distribution is influenced by genetic factors. For instance, in humans, waisthip ratio, a measure of body fat distribution independent of overall adiposity, is a heritable trait controlled by multiple significant loci (Heid et al., 2010). Recently, Melendez et al. (2018) provided the first genetic report for visceral fat accumulation in dairy cows. These authors found that visceral fat deposition is a heritable trait, and hence, its prevalence in dairy cattle could be reduced by genetic selection. They also found evidence that variation in genes involved in preadipocyte signaling and adipocyte development could explain part of the differences observed in visceral fat accumulation.

The first objective of this study was to reanalyze the data from Melendez et al. (2018) using alternative methods for gene mapping and the application of SNP-based gene-set enrichment tools. Given that visceral fat accumulation is associated with metabolic health, the second objective of this study was to identify genomic regions with pleotropic effects on visceral adiposity and incidence of ketosis or displaced abomasum.

\section{MATERIALS AND METHODS}

\section{Visceral Fat Accumulation}

Data were collected at a slaughterhouse located in Green Bay, WI, United States (Melendez et al., 2018). Briefly, adult Holstein cows were evaluated from the processing line. After the carcass was eviscerated, the amount of omental fat at the level of the insertion of the lesser omentum over the pylorus area was assessed. Low visceral fat accumulation was defined as an omental fold $<5 \mathrm{~mm}$ in thickness and minimum fat deposition observed throughout the entire omentum. High visceral fat accumulation was defined as an omental fold $\geq 20 \mathrm{~mm}$ in thickness and marked fat deposition observed throughout the entire omentum. Body condition score was measured using a 5-point scale, and only cows with body condition score between 2.75 and 3.25 were considered. As mentioned by Melendez et al. (2018), the goal of this sampling protocol was to obtain two groups of Holstein cows with extreme differences in visceral fat accumulation but with very similar subcutaneous fat deposition. A total of 172 cows were finally selected for this study, 77 with low and 95 with high visceral fat accumulation.

These 172 cows were genotyped using the Illumina BovineHD Beadchip with over 777k single nucleotide polymorphism (SNP) probes. After removing monomorphic markers and those located in the X chromosome, a total of 584,557 SNPs remained for the genomic analyses.

\section{Genomic Scans}

The importance of additive and non-additive effects on visceral fat accumulation was evaluated using a two-step mixed-modelbased approach (Aulchenko et al., 2007).

In the first step, the following model was fitted:

$$
\mathbf{y}=\mathbf{X b}+\mathbf{Z u}+\mathbf{e}
$$

where $\mathbf{y}$ is the vector of visceral fat accumulation scores (low $=0$ or high =1), $\mathbf{b}$ is the vector of fixed effects, $\mathbf{u}$ is the vector of random animal effects, and $\mathbf{e}$ is the vector of random residual effects. The incidence matrices $\mathbf{X}$ and $\mathbf{Z}$ relate visceral fat accumulation scores to fixed and animal effects, respectively. The random effects were assumed to follow a multivariate normal distribution with $\mathbf{u} \sim N\left(0, \mathbf{G} \sigma_{u}^{2}\right)$ and $\mathbf{e} \sim N\left(0, \mathbf{I} \sigma_{e}^{2}\right)$, where $\sigma_{u}^{2}$ and $\sigma_{e}^{2}$ are the animal additive genetic and residual variances respectively, $\mathbf{G}$ is the genomic relationship matrix, and $\mathbf{I}$ an identity matrix. The variance-covariance matrix for this first model was estimated as $\mathbf{V}_{0}=\mathbf{Z} \mathbf{G Z} \mathbf{\sigma}_{u}^{2}+\mathbf{I} \sigma_{e}^{2}$.

In the second step, the following model was fitted for every SNP:

$$
\mathrm{y}=\mathbf{X} \boldsymbol{\beta}+X_{S N P} \beta_{S N P}+\epsilon
$$

where $X_{S N P}$ is the design matrix for the marker under consideration and $\beta_{S N P}$ is the regression coefficient, also known as SNP effect. Every SNP genotype was coded using single numeric variables as $(0,1,2),(0,1,1),(0,0,1)$ and $(0$, $1,0)$ for testing additive, dominance, recessive and overdominance effects, respectively. This model assumes that $\epsilon \sim N\left(0, \mathbf{V}_{0} \sigma_{e}^{2}\right)$. The significance of each SNP effect was evaluated using the following test statistic:

$$
\mathbf{z}=\frac{\mathbf{X}_{S N P}^{\prime} \mathbf{V}_{0}^{-1}(\mathbf{y}-\mathbf{X} \hat{\boldsymbol{\beta}})}{\sqrt{\mathbf{X}_{S N P}^{\prime} \boldsymbol{V}_{0}^{-1} \mathbf{X}_{S N P}}}
$$


Which approximates the Wald test, and hence, is asymptotically standard normal. These analyses were performed using the $\mathrm{R}$ package MixABEL (Aulchenko et al., 2007). The possible inflation of the test statistics was evaluated using quantile-quantile (Q-Q) plots.

\section{Overrepresentation Analysis}

The overrepresentation analysis, also known as gene-set analysis, was performed in three steps as described by Han and Peñagaricano (2016). The first step was the assignment of SNP markers to annotated genes. The latest bovine reference annotation (ARS-UCD1.2) was used to retrieve the exact location of each annotated bovine gene in the genome. SNP markers were assigned to annotated genes if they were located within the genomic sequence of the gene or at most $10 \mathrm{~kb}$ upstream or downstream the gene. Significant genes were defined as those genes containing at least one SNP with a significant additive effect ( $p$-value $\leq 0.01)$. The second step was the assignment of annotated genes to gene-sets. Six different gene-set databases were explored, including GO, KEGG, MeSH, InterPro, MSigDB, and Reactome. Finally, in the third step, the enrichment or overrepresentation of significant genes in each gene-set was tested using a Fisher's exact test. All these analyses were performed using the $R$ package EnrichKit (https://github.com/liulihe954/EnrichKit), developed by our group.

\section{Genomic Analysis of Metabolic Disorders}

Data consisted of producer-recorded lactation incidence records of two important metabolic disorders, namely displaced abomasum and ketosis, collected in one large commercial dairy herd in the state of Florida, USA. A total of 27,408 records of displaced abomasum from 13,401 cows and 26,752 records of ketosis from 13,133 cows were collected between January 2010 and December 2015. Metabolic disorders were recorded as binary, i.e., $\mathrm{Y}=1$ if the cow had clinical symptoms, and $\mathrm{Y}=0$ otherwise. A total of 487 and 2393 cows had at least one case of displaced abomasum or ketosis, respectively. The incidence of displaced abomasum was $1.8 \%$ (491 cases) while the incidence of ketosis was $10.4 \%$ (2772 cases). Genotype data for 60,671 single nucleotide polymorphism (SNP) markers were available for $5.9 \mathrm{k}$ cows with health records and $1.4 \mathrm{k}$ sires in the pedigree. Markers that mapped to the sex chromosomes, or were monomorphic, or had minor allele frequency less than $1 \%$ were removed from the SNP dataset. After data editing, a total of 54,043 SNPs were retained for subsequent analyses.

The incidence of metabolic disorders was analyzed using a threshold model (Gianola, 1982). This model, also known as probit model, describes the observable response variable ( 0 or 1 ) using an underlying linear model, $\mathbf{z}=\boldsymbol{\eta}+\boldsymbol{\varepsilon}$, where $\boldsymbol{\eta}$ is a vector of linear predictors and $\varepsilon$ is a vector of independent and identically distributed standard normal random variables. Here, the liner predictor $\boldsymbol{\eta}$ had the following form:

$$
\eta=X \boldsymbol{\beta}+Z_{1} \text { hys }+Z_{2} \mathbf{u}+\mathbf{W p e}
$$

where $\boldsymbol{\beta}$ is a vector of fixed effects in the model, hys is a vector of random herd-year-season effects, $\mathbf{u}$ is a vector of random additive genetic effects, and pe is a vector of random permanent environmental effects. The vector $\boldsymbol{\beta}$ includes the intercept and the lactation number as a class variable with 5 levels $(1,2,3,4$, and $5+)$. The matrices $\mathbf{X}, \mathbf{Z}_{1}, \mathbf{Z}_{2}$, and $\mathbf{W}$ are the incidence matrices relating health records to fixed, herd-year-season, animal, and permanent environmental effects, respectively. The random effects were assumed to follow a multivariate normal distribution with hys $\sim N\left(0, \mathbf{I} \sigma_{h y s}^{2}\right), \quad \mathbf{u} \sim N\left(0, \mathbf{H} \sigma_{u}^{2}\right)$, and pe $\sim N\left(0, \mathbf{I} \sigma_{p e}^{2}\right)$, where $\sigma_{h y s}^{2}, \sigma_{u}^{2}$ and $\sigma_{p e}^{2}$ are the hear-yearseason, animal additive genetic, and permanent environmental variances respectively, $\mathbf{H}$ is a relationship matrix, and $\mathbf{I}$ an identity matrix. The matrix $\mathbf{H}$ combines pedigree and genotypic information (Aguilar et al., 2010).

Candidate genomic regions associated with metabolic disorders were identified based on the amount of genetic variance explained by $2.0 \mathrm{Mb}$ window of adjacent SNPs. The SNP effects were estimated as $\hat{\mathbf{s}}=\mathbf{D} \mathbf{M}^{\prime}\left[\mathbf{M D M}^{\prime}\right]^{-1} \hat{\mathbf{a}}_{\mathbf{g}}$, where $\hat{\mathbf{s}}$ is the vector of SNP marker effects, $\mathbf{D}$ is a diagonal matrix of weights of SNPs, $\mathbf{M}$ is a matrix relating genotypes of each SNP marker to observations, and $\hat{\mathbf{a}}_{\mathbf{g}}$ is the vector of genomic estimated breeding values for genotyped animals (Wang et al., 2012). The percentage of genetic variance explained by a $2.0 \mathrm{Mb}$ region was calculated as,

$$
\frac{\operatorname{Var}\left(u_{i}\right)}{\sigma_{u}^{2}} \times 100=\frac{\operatorname{Var}\left(\sum_{j=1}^{N} M_{j} s_{j}\right)}{\sigma_{u}^{2}} \times 100
$$

where $u_{i}$ is the genetic value of the $i^{\text {th }}$ genomic region under consideration, $N$ is the total number of adjacent SNPs within the 2.0 Mb region, $M_{j}$ is the vector of SNP content of the $j^{\text {th }}$ SNP for all individuals, and $s_{j}$ is the marker effect of the $j^{\text {th }}$ SNP within the $i^{t h}$ region. These analyses were performed using the program POSTGSF90 (Aguilar et al., 2014).

\section{RESULTS}

\section{Genomic Scans for Visceral Fat Accumulation}

Figure 1 shows the results of the whole-genome single marker scans for testing both additive and non-additive (recessive) genetic effects on visceral fat accumulation in Holstein cows. Genomic regions on chromosomes BTA19, BTA20 and BTA24 showed the most significant additive effects (Table 1). The two significant regions in BTA19 (10.32-10.62 Mb and $37.89-38.19 \mathrm{Mb}$ ) harbor candidate genes DHX40, YPEL2, CLTC, SKAP1, and HOXB1-6 family. These genes are implicated in different functions, including cell proliferation, cell differentiation and immunity. Moreover, the significant region detected in BTA20 $(22.88-23.18 \mathrm{Mb})$ harbors the gene $A N K R D 55$, which is related to adipocyte proliferation and lipolysis. Finally, the significant region in BTA24 $(48.46-48.76 \mathrm{Mb})$ harbors the gene SMAD7, which is associated with glucose uptake and obesity. 


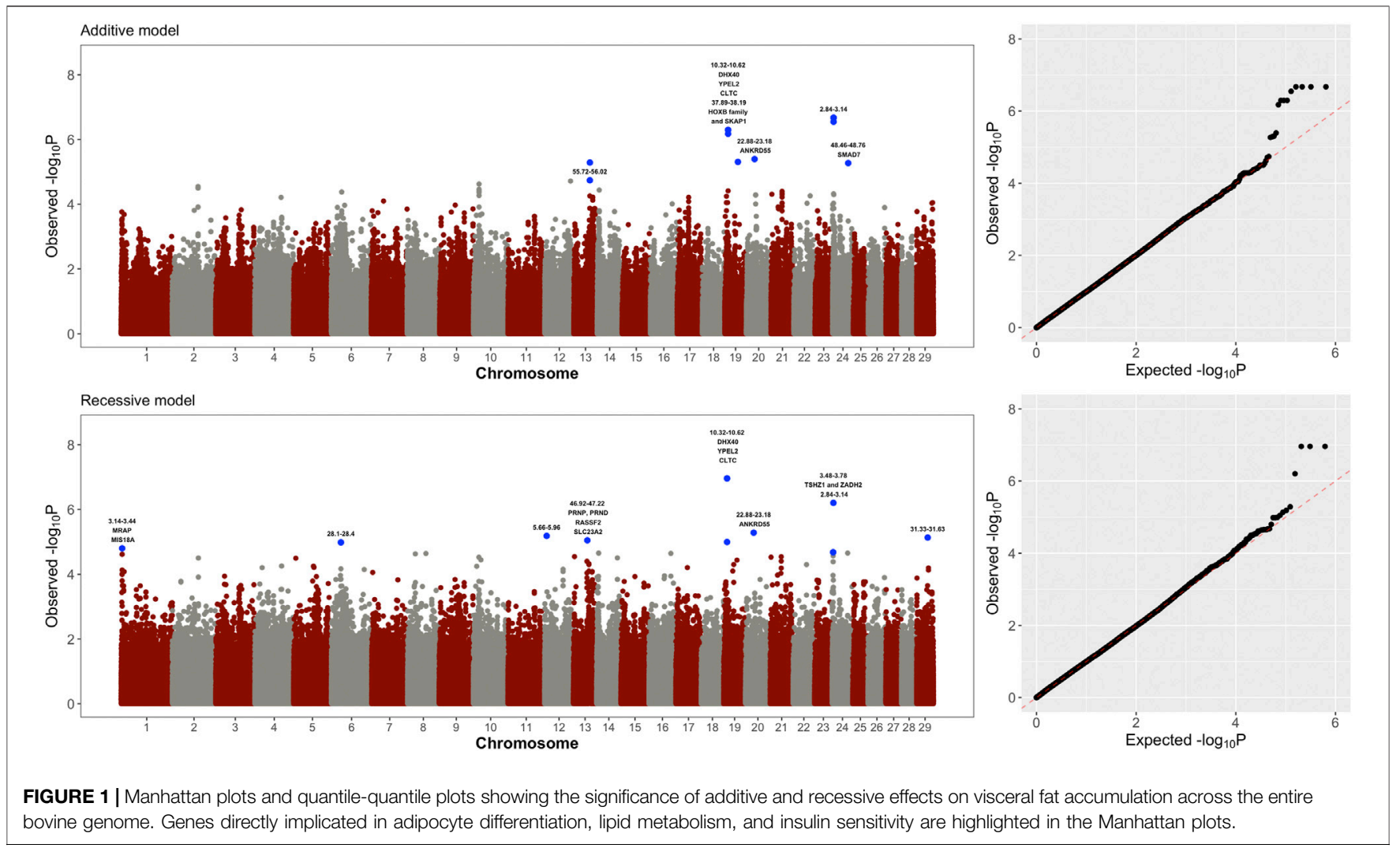

TABLE 1 | Genetic markers and candidate genes associated with visceral fat accumulation in Holstein cows

\begin{tabular}{|c|c|c|c|c|}
\hline Chromosome & Position & $p$-value & Candidate gene & Process \\
\hline \multicolumn{5}{|c|}{ Markers with additive effects } \\
\hline BTA19 & $10.32-10.62$ & $5.0 \mathrm{e}-07$ & YPEL2 & Cell cycle \\
\hline \multirow[t]{3}{*}{ BTA19 } & $37.89-38.19$ & $4.9 \mathrm{e}-06$ & CLTC & Cell proliferation \\
\hline & & $4.9 e-06$ & HOXB1-6 & Development, morphogenesis \\
\hline & & $4.9 e-06$ & SKAP1 & Adaptive immune response \\
\hline BTA20 & $22.88-23.18$ & 4.0e-06 & ANKRD55 & Pre-adipocyte proliferation, differentiation \\
\hline BTA24 & $48.46-48.76$ & $5.4 \mathrm{e}-06$ & SMAD7 & Regulation of TGF- $\beta$ signaling \\
\hline \multicolumn{5}{|c|}{ Markers with non-additive effects } \\
\hline \multirow[t]{2}{*}{ BTA1 } & $3.14-3.39$ & $2.4 \mathrm{e}-05$ & MRAP & Lipolysis, energy balance \\
\hline & & $2.4 \mathrm{e}-05$ & MIS18A & Cell cycle, cell proliferation \\
\hline \multirow[t]{2}{*}{ BTA13 } & $46.92-47.22$ & $8.9 e-06$ & PRNP & Adipocyte differentiation \\
\hline & & $8.9 \mathrm{e}-06$ & SLC23A2 & Vitamin C transport \\
\hline \multirow[t]{2}{*}{ BTA24 } & $3.48-3.78$ & $6.3 e-07$ & TSHZ1 & Pancreatic $\beta$-cell maturation \\
\hline & & $6.3 e-07$ & $\mathrm{ZADH} 2$ & Adipocyte differentiation \\
\hline
\end{tabular}

Of particular interest, three genomic regions located in BTA1, BTA13 and BTA24 showed purely non-additive (recessive) effects (Figure $\mathbf{1}$ and Table 1). The region in BTA1 (3.14-3.39 Mb) harbors the candidate genes MRAP and MIS18A that are implicated in insulin sensitivity and obesity. The region in BTA13 (46.92-47.22 Mb) harbors the gene PRNP which is directly involved in visceral fat adipose tissue deposition. The significant region in BTA24 $(3.48-3.78 \mathrm{Mb})$ harbors the gene TSHZ1 and ZADH2 that is associated with adipocyte differentiation and lipid metabolism.

\section{Overrepresentation Analysis}

The overrepresentation analysis, namely the search for gene-sets or gene pathways that show an overrepresentation of significant genes, was performed using a Fisher's exact test, a test of proportions based on the cumulative hypergeometric distribution. Figure 2 shows a set of terms that were significantly enriched with genes associated with visceral fat accumulation. These functional terms are related to calcium signaling, cell arrangement, cell metabolism, cell proliferation, cell signaling, immune response, lipid metabolism, membrane permeability, and nervous signaling, among other functions. 


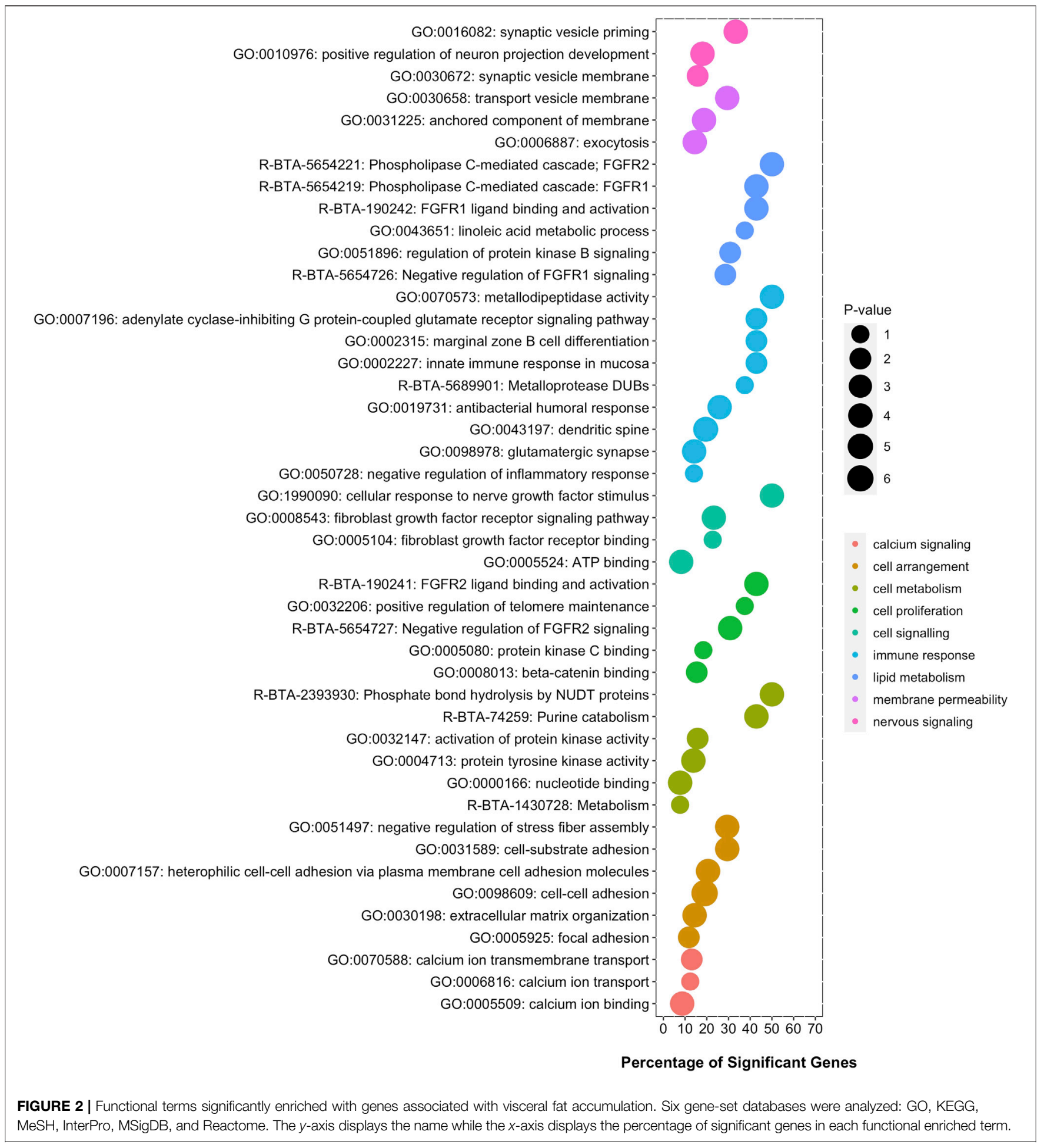

\section{Genomic Analysis of Metabolic Disorders}

The identification of genomic regions affecting displaced abomasum or ketosis was performed using the single-step genomic BLUP. This method combines all the available phenotypic, genotypic, and pedigree information, and fits all the SNP simultaneously. Candidate regions were identified based on the amount of genetic variance explained by $2.0 \mathrm{Mb} \mathrm{SNP}$-windows. Figure 3 shows the gene mapping results for displaced abomasum. Notably, the prominent peak in BTA20, which harbors gene ANKRD55, was also significantly associated with visceral fat accumulation, suggesting a pleiotropic action. On the other hand, there were not common regions between ketosis and visceral fat accumulation (data not shown). 


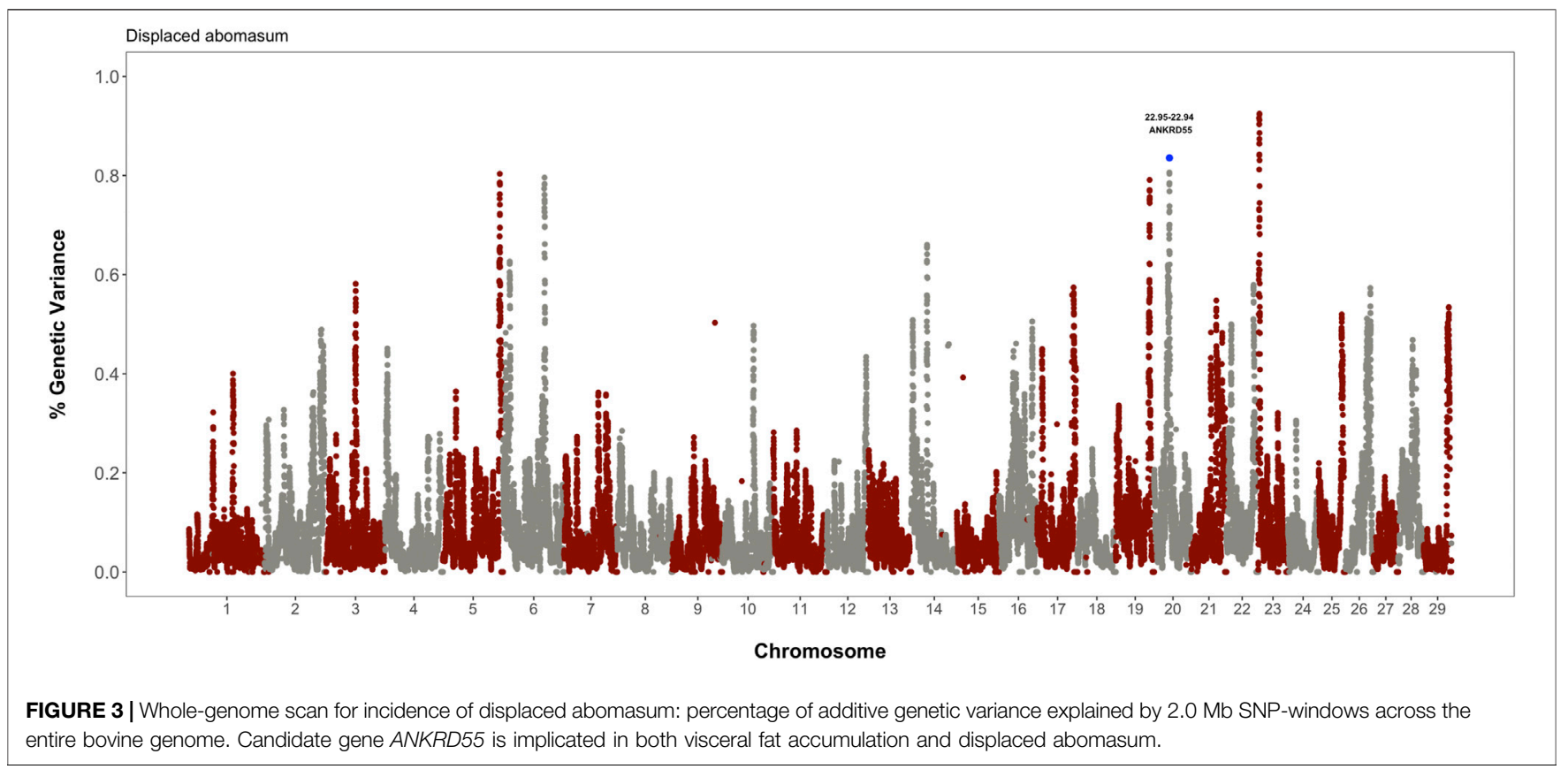

\section{DISCUSSION}

Visceral fat is a highly active tissue involved in complex metabolic processes, such as energy supply, inflammation, and insulin sensitivity. Dairy cows with excessive visceral fat are more susceptible to metabolic disorders (Drackley et al., 2014; Ji et al., 2014a; Singh et al., 2014). Although its importance on health and production traits, very few studies have investigated the genetic basis of visceral fat accumulation in dairy cattle. As such, this study was specially conducted to identify individual genes, functional gene-sets and biological pathways associated with visceral fat accretion in Holstein cows. Two groups of cows with extreme differences in fat deposition in the omentum, one of the most important visceral fat depots, but with very similar subcutaneous fat deposition, were evaluated. Note that omental fat is strongly correlated with total visceral fat. Furthermore, given the relationship between visceral fat and metabolic disorders, we also investigated the genetic link between visceral fat accumulation and the incidence of displaced abomasum and ketosis in early lactation.

Several pathways revealed in our study coincide with cell rearrangement of adipose tissue identified in other species in obesity studies. In fact, our gene-set analysis detected biological pathways directly involved in the visceral fat expansion, such as cell arrangement, cell proliferation and cytoskeleton regulation. Additionally, some of the most significant genes detected in our whole-genome scans are directly implicated in lipid accumulation and tissue rearrangement. For instance, gene PRNP encodes the cellular prior protein known to regulate visceral fat volume, body fat weight, adipocyte cell size, and body weight gain in mice (Jeong et al., 2019). Similarly, the significant gene $Z A D H 2$, also known as PTGR-3, negatively modulates adipocyte differentiation through regulation of $\operatorname{PPAR} \gamma$, a major regulator of adipogenesis (Yu et al., 2013). Regarding cell rearrangement, the significant gene YPEL2 is known to be involved in cell division (Hosono et al., 2004) and the HOXB family to encode for transcription factors involved in the anatomical structure of omental fat (Ahn et al., 2019). Genes implicated in the cascade signaling due to the active release of NEFA on liver were also detected. Notably, gene $S M A D 7$, one of the most significant genes revealed in the association mapping, is associated with higher levels of circulating NEFA, lower expression of lipolytic genes, and more proinflammatory proteins in obese mice (Seong et al., 2018). While the action of SMAD7 occurs by downregulating TGF- $\beta$ pathway, the significant gene CLTC stimulates this pathway while downregulate NADPH oxidase to protect against the negative effects of highly active NEFA oxidation (Han, 2016; Caballero-Díaz et al., 2020). Additionally, our overrepresentation analysis detected pathways related to fibroblast growth factor receptors (FGFR), calcium signaling, protein kinases and glutamate signaling. The FGFR1 signaling pathway is related to lipid droplet dynamics, phospholipid homeostasis, protection against oxidative stress and to hypertrophy in obese individuals (Ye et al., 2016). The FGFR2 signaling pathway, inhibited by the significant gene SMAD7, indirectly promotes lipid biosynthesis by reducing cAMP pool and protein kinase A (PKA) activity (Ornitz and Itoh, 2015; Huang et al., 2018). Interestingly, both cAMP and PKA are affected by the gene $M R A P$, a significant gene detected in the non-additive scan. Of particular interest, this gene is associated with mitochondrial fatty acid oxidation and is essential for the lipolytic response to adrenocorticoid hormone, and consequently, insulin sensitivity (Zhang et al., 2018). 
Research has shown that inflammation is directly impacted by higher availability of glucose and NEFA (Patel and Abate, 2013). Interestingly, our study revealed many genes and gene-sets associated with inflammation. For instance, the gene NOX4, the main source of reactive oxygen species (ROS), is highly expressed in adipocytes and is controlled by gene CLTC, which was detected as significantly associated with fat accumulation in our whole-genome scan (Den Hartigh et al., 2017). Our enrichment analysis detected calcium transport terms that are known to be affected by ROS in the form of impaired calcium homeostasis that can lead to cell death (Dejos et al., 2020). Other adipose-related inflammation responses were also detected is this work. For instance, the significant gene SLC23A2 codes for SVCT1, a transporter of vitamin C, a well-known antioxidant that is able to inhibit adipocyte differentiation and lipid accumulation (Rahman et al., 2014). Significant functional terms such as protein kinase $\mathrm{C}$, dendritic spine, and metalloproteinases may indicate the response to local inflammation via proliferation, activation and communication of T-cells, respectively (Black and Black, 2012; Khokha et al., 2013; Sundara Rajan and Longhi, 2016). Interestingly, the gene SKAP1 identified in the genomic scan is an immune cell adaptor responsible for regulating multiple functions of T-cells (Raab et al., 2019). Enriched terms such as metalloproteases and bacterial humoral defense can also be related to systemic inflammation as the threshold between the beneficial acute inflammation and damaging effect of chronic inflammation is controlled by metalloproteinases via macrophage activity (Khokha et al., 2013).

There is growing evidence that excessive visceral fat may lead to metabolic disorders. In fact, the significant genes and pathways identified in this study suggest a differential inflammatory response among cows with different levels of visceral fat. It is known that cows with displaced abomasum have preferable mobilization of visceral over subcutaneous fat and present higher macrophage infiltration in the omental adipose tissue compared to healthy individuals (Hostens et al., 2012; Contreras et al., 2015). Interestingly, our work revealed one region in chromosome 20 that has significant effects on both visceral fat accumulation and susceptibility to displaced abomasum. Notably, this region harbors the gene ANKRD55, which encodes a scaffold protein related to proliferation of pre-adipocytes, insulin sensitivity, and even more important, higher visceral fat accumulation in human subjects (Harder et al., 2013; Ji et al., 2019; Chen et al., 2020). Gene ANKRD55 is highly active in immune diseases, which corresponds to the state of the clinically diagnosed displaced abomasum cows used in this study, as studies have shown that cows with displaced abomasum are under active lipolysis, negative energy balance, and under higher infiltration of macrophages in adipose tissues (Hostens et al., 2012; Contreras et al., 2015). The whole-body insulin resistance possibly promoted by $A N K R D 55$ would endorse the insulin resistance stimulated by cytokines release from visceral fat and the impaired glucose-stimulated insulin secretion in pancreatic $\beta$ cells mediated by the significant gene TSHZ1 (Raum et al., 2015). Changes in insulin concentration and blood calcium levels, two mechanisms identified in our gene-set analysis, are some of the causes for displaced abomasum in cows (Van Winden et al., 2003). This common peak in BTA20 for visceral fat accumulation and displaced abomasum suggests a genetic link between visceral fat levels and the incidence of metabolic diseases that deserves further investigation.

\section{CONCLUSION}

In this study, we performed an integrative genomic analysis to better understand the genetic basis of visceral fat accumulation in Holstein cows. Our analysis revealed several genomic regions with significant additive and non-additive genetic effects. Interestingly, these regions harbor genes, such as $S M A D 7$, SKAP1, ANKRD55, MRAP and MIS18A, that are directly implicated in adipocyte differentiation, lipid metabolism, immune response, and insulin tolerance. We also performed a gene-set analysis to gain additional insights into the genetic architecture of visceral fat deposition. Our analysis revealed gene pathways and molecular mechanisms related to cell metabolism, cell signaling, and immune response, among others. We also assessed the genetic link between visceral fat and metabolic disorders. Notably, one region on BTA20, which harbors the gene ANKRD55, implicated in adipocyte differentiation and insulin resistance, showed significant effects on both visceral fat accumulation and displaced abomasum. Overall, our study suggests that visceral fat deposition in dairy cows is controlled by both additive and non-additive genetic factors. The genetic link between visceral fat accumulation and metabolic disorders deserves further investigation.

\section{DATA AVAILABILITY STATEMENT}

The original contributions presented in the study are included in the article/Supplementary Material, further inquiries can be directed to the corresponding author.

\section{ETHICS STATEMENT}

The animal study was reviewed and approved by University of Missouri, protocol number 8443 .

\section{AUTHOR CONTRIBUTIONS}

FP, PM, and PP designed the study. PM performed the field study. LN, LC, and FP analyzed the data. LN, LC, PP, PM, and FP contributed to the interpretation of the results. LN wrote the first draft of the manuscript. All authors have read and approved this manuscript.

\section{ACKNOWLEDGMENTS}

LN was supported by the Fulbright Brazil Commission and Coordenação de Aperfeiçoamento de Pessoal de Nível Superior - Brasil (CAPES) - Finance Code 001. 


\section{REFERENCES}

Aguilar, I., Misztal, I., Johnson, D. L., Legarra, A., Tsuruta, S., and Lawlor, T. J. (2010). Hot Topic: A Unified Approach to Utilize Phenotypic, Full Pedigree, and Genomic Information for Genetic Evaluation of Holstein Final Score. J. Dairy Sci. 93, 743-752. doi:10.3168/jds.2009-2730

Aguilar, I., Misztal, I., Tsuruta, S., and Legarra, A. (2014). PREGSF90 POSTGSF90: Computational Tools for the Implementation of Single-step Genomic Selection and Genome-wide, in Proceedings, 10th World Congress of Genetics Applied to Livestock Production. (Vancouver, BC, Canada: American Society of Animal Science), 0-3. doi:10.13140/2.1.4801.5045

Ahn, J., Wu, H., and Lee, K. (2019). Integrative Analysis Revealing Human Adipose-specific Genes and Consolidating Obesity Loci. Sci. Rep. 9, 1-13. doi:10.1038/s41598-019-39582-8

Aulchenko, Y. S., de Koning, D. J., and Haley, C. (2007). Genomewide Rapid Association Using Mixed Model and Regression: A Fast and Simple Method for Genomewide Pedigree-Based Quantitative Trait Loci Association Analysis. Genetics 177, 577-585. doi:10.1534/genetics.107.075614

Black, A. R., and Black, J. D. (2012). Protein Kinase C Signaling and Cell Cycle Regulation. Front. Immun. 3, 1-17. doi:10.3389/fimmu.2012.00423

Booth, A., Magnuson, A., Fouts, J., and Foster, M. T. (2016). Adipose Tissue: An Endocrine Organ Playing a Role in Metabolic Regulation. Horm. Mol. Biol. Clin. Investig. 26, 25-42. doi:10.1515/hmbci-2015-0073

Caballero-Díaz, D., Bertran, E., Peñuelas-Haro, I., Moreno-Càceres, J., Malfettone, A., López-Luque, J., et al. (2020). Clathrin Switches Transforming Growth Factor- $\beta$ Role to Pro-tumorigenic in Liver Cancer. J. Hepatol. 72, 125-134. doi:10.1016/j.jhep.2019.09.012

Chen, Z., Yu, H., Shi, X., Warren, C. R., Lotta, L. A., Friesen, M., et al. (2020). Functional Screening of Candidate Causal Genes for Insulin Resistance in Human Preadipocytes and Adipocytes. Circ. Res. 126, 330-346. doi:10.1161/ CIRCRESAHA.119.315246

Contreras, G. A., Kabara, E., Brester, J., Neuder, L., and Kiupel, M. (2015). Macrophage Infiltration in the Omental and Subcutaneous Adipose Tissues of Dairy Cows with Displaced Abomasum. J. Dairy Sci. 98, 6176-6187. doi:10.3168/jds.2015-9370

Dejos, C., Gkika, D., and Cantelmo, A. R. (2020). The Two-Way Relationship between Calcium and Metabolism in Cancer. Front. Cell Dev. Biol. 8, 1-14. doi:10.3389/fcell.2020.573747

Den Hartigh, L. J., Omer, M., Goodspeed, L., Wang, S., Wietecha, T., O’Brien, K. D., et al. (2017). Adipocyte-Specific Deficiency of NADPH Oxidase 4 Delays the Onset of Insulin Resistance and Attenuates Adipose Tissue Inflammation in Obesity. Atvb 37, 466-475. doi:10.1161/ATVBAHA.116.308749

Drackley, J. K., Wallace, R. L., Graugnard, D., Vasquez, J., Richards, B. F., and Loor, J. J. (2014). Visceral Adipose Tissue Mass in Nonlactating Dairy Cows Fed Diets Differing in Energy Density1. J. Dairy Sci. 97, 3420-3430. doi:10.3168/jds.20148014

Gianola, D. (1982). Theory and Analysis of Threshold Characters. J. Anim. Sci. 54, 1079-1096. doi:10.2527/jas1982.5451079x

Han, C. Y. (2016). Roles of Reactive Oxygen Species on Insulin Resistance in Adipose Tissue. Diabetes Metab. J. 40, 272-279. doi:10.4093/dmj.2016.40.4.272

Han, Y., and Peñagaricano, F. (2016). Unravelling the Genomic Architecture of Bull Fertility in Holstein Cattle. BMC Genet. 17, 143. doi:10.1186/s12863-0160454-6

Harder, M. N., Ribel-Madsen, R., Justesen, J. M., Sparsø, T., Andersson, E. A., Grarup, N., et al. (2013). Type 2 Diabetes Risk Alleles Near BCAR1 and in ANK1 Associate with Decreased $\beta$-Cell Function whereas Risk Alleles Near ANKRD55 and GRB14 Associate with Decreased Insulin Sensitivity in the Danish Inter99 Cohort. J. Clin. Endocrinol. Metab. 98, E801-E806. doi:10.1210/ jc.2012-4169

Heid, I. M., Jackson, A. U., Randall, J. C., Winkler, T. W., Qi, L., Steinthorsdottir, V., et al. (2010). Meta-analysis Identifies 13 New Loci Associated with WaistHip Ratio and Reveals Sexual Dimorphism in the Genetic Basis of Fat Distribution. Nat. Genet. 42, 949-960. doi:10.1038/ng.685

Hosono, K., Sasaki, T., Minoshima, S., and Shimizu, N. (2004). Identification and Characterization of a Novel Gene Family YPEL in a Wide Spectrum of Eukaryotic Species. Gene 340, 31-43. doi:10.1016/j.gene.2004.06.014
Hostens, M., Fievez, V., Leroy, J. L. M. R., Van Ranst, J., Vlaeminck, B., and Opsomer, G. (2012). The Fatty Acid Profile of Subcutaneous and Abdominal Fat in Dairy Cows with Left Displacement of the Abomasum. J. Dairy Sci. 95, 3756-3765. doi:10.3168/jds.2011-5092

Huang, X., Liu, G., Guo, J., and Su, Z. (2018). The PI3K/AKT Pathway in Obesity and Type 2 Diabetes. Int. J. Biol. Sci. 14, 1483-1496. doi:10.7150/ijbs.27173

Item, F., and Konrad, D. (2012). Visceral Fat and Metabolic Inflammation: The portal Theory Revisited. Obes. Rev. 13, 30-39. doi:10.1111/j.1467789X.2012.01035.x

Jeong, J.-K., Lee, J.-H., Kim, S.-W., Hong, J.-M., Seol, J.-W., and Park, S.-Y. (2019). Cellular Prion Protein Regulates the Differentiation and Function of Adipocytes through Autophagy Flux. Mol. Cell Endocrinol. 481, 84-94. doi:10.1016/j.mce.2018.11.013

Ji, P., Drackley, J. K., Khan, M. J., and Loor, J. J. (2014a). Inflammation- and Lipid Metabolism-Related Gene Network Expression in Visceral and Subcutaneous Adipose Depots of Holstein Cows. J. Dairy Sci. 97, 3441-3448. doi:10.3168/ jds.2013-7296

Ji, P., Drackley, J. K., Khan, M. J., and Loor, J. J. (2014b). Overfeeding Energy Upregulates Peroxisome Proliferator-Activated Receptor (PPAR) $\gamma$-controlled Adipogenic and Lipolytic Gene Networks but Does Not Affect Proinflammatory Markers in Visceral and Subcutaneous Adipose Depots of Holstein Cows. J. Dairy Sci. 97, 3431-3440. doi:10.3168/jds.2013-7295

Ji, Y., Yiorkas, A. M., Frau, F., Mook-Kanamori, D., Staiger, H., Thomas, E. L., et al. (2019). Genome-wide and Abdominal MRI Data Provide Evidence that a Genetically Determined Favorable Adiposity Phenotype Is Characterized by Lower Ectopic Liver Fat and Lower Risk of Type 2 Diabetes, Heart Disease, and Hypertension. Diabetes 68, 207-219. doi:10.2337/db18-0708

Khokha, R., Murthy, A., and Weiss, A. (2013). Metalloproteinases and Their Natural Inhibitors in Inflammation and Immunity. Nat. Rev. Immunol. 13 649-665. doi:10.1038/nri3499

Konrad, D., and Wueest, S. (2014). The Gut-Adipose-Liver axis in the Metabolic Syndrome. Physiology 29, 304-313. doi:10.1152/physiol.00014.2014

Melendez, P., Poock, S. E., Pithua, P., Pinedo, P., Manriquez, D., Moore, S. G., et al. (2019). Genome-wide Study to Detect Single Nucleotide Polymorphisms Associated with Visceral and Subcutaneous Fat Deposition in Holstein Dairy Cows. Animal 13, 487-494. doi:10.1017/S1751731118001519

Ornitz, D. M., and Itoh, N. (2015). The Fibroblast Growth Factor Signaling Pathway. Wires Dev. Biol. 4, 215-266. doi:10.1002/wdev.176

Pascottini, O. B., Leroy, J. L. M. R., and Opsomer, G. (2020). Metabolic Stress in the Transition Period of Dairy Cows: Focusing on the Prepartum Period. Animals 10, 1419. doi:10.3390/ani10081419

Patel, P., and Abate, N. (2013). Body Fat Distribution and Insulin Resistance. Nutrients 5, 2019-2027. doi:10.3390/nu5062019

Raab, M., Strebhardt, K., and Rudd, C. E. (2019). Immune Adaptor SKAP1 Acts a Scaffold for Polo-like Kinase 1 (PLK1) for the Optimal Cell Cycling of T-Cells. Sci. Rep. 9, 1-13. doi:10.1038/s41598-019-45627-9

Rahman, F., Al Frouh, F., Bordignon, B., Fraterno, M., Landrier, J.-F. o., Peiretti, F., et al. (2014). Ascorbic Acid Is a Dose-dependent Inhibitor of Adipocyte Differentiation, Probably by Reducing cAMP Pool. Front. Cell Dev. Biol. 2, 1-10. doi:10.3389/fcell.2014.00029

Raum, J. C., Soleimanpour, S. A., Groff, D. N., Coré, N., Fasano, L., Garratt, A. N., et al. (2015). Tshz1 Regulates Pancreatic $\beta$-Cell Maturation. Diabetes 64, 2905-2914. doi:10.2337/db14-1443

Seong, H.-A., Manoharan, R., and Ha, H. (2018). Smad Proteins Differentially Regulate Obesity-Induced Glucose and Lipid Abnormalities and Inflammation via Class-specific Control of AMPK-Related Kinase MPK38/MELK Activity. Cell Death Dis 9, 471. doi:10.1038/s41419-018-0489-x

Singh, S. P., Häussler, S., Heinz, J. F. L., Akter, S. H., Saremi, B., Müller, U., et al. (2014). Lactation Driven Dynamics of Adiponectin Supply from Different Fat Depots to Circulation in Cows. Domest. Anim. Endocrinol. 47, 35-46. doi:10.1016/j.domaniend.2013.12.001

Sundara Rajan, S., and Longhi, M. P. (2016). Dendritic Cells and Adipose Tissue. Immunology 149, 353-361. doi:10.1111/imm.12653

Van Winden, S. C. L., Jorritsma, R., Müller, K. E., and Noordhuizen, J. P. T. M. (2003). Feed Intake, Milk Yield, and Metabolic Parameters Prior to Left Displaced Abomasum in Dairy Cows. J. Dairy Sci. 86, 1465-1471. doi:10.3168/jds.S0022-0302(03)73730-8 
Wang, H., Misztal, I., Aguilar, I., Legarra, A., and Muir, W. M. (2012). Genome-wide Association Mapping Including Phenotypes from Relatives without Genotypes. Genet. Res. 94, 73-83. doi:10.1017/ S0016672312000274

Ye, M., Lu, W., Wang, X., Wang, C., Abbruzzese, J. L., Liang, G., et al. (2016). FGF21-FGFR1 Coordinates Phospholipid Homeostasis, Lipid Droplet Function, and ER Stress in Obesity. Endocrinology 157, 4754-4769. doi:10.1210/en.2016-1710

Yu, Y.-H., Chang, Y.-C., Su, T.-H., Nong, J.-Y., Li, C.-C., and Chuang, L.-M. (2013). Prostaglandin Reductase-3 Negatively Modulates Adipogenesis through Regulation of PPAR $\gamma$ Activity. J. Lipid Res. 54, 2391-2399. doi:10.1194/jlr.M037556

Zhang, X., Saarinen, A. M., Campbell, L. E., De Filippis, E. A., and Liu, J. (2018). Regulation of Lipolytic Response and Energy Balance by Melanocortin 2 Receptor Accessory Protein (MRAP) in Adipocytes. Diabetes 67, 222-234. doi:10.2337/db17-0862
Conflict of Interest: The authors declare that the research was conducted in the absence of any commercial or financial relationships that could be construed as a potential conflict of interest.

Publisher's Note: All claims expressed in this article are solely those of the authors and do not necessarily represent those of their affiliated organizations, or those of the publisher, the editors and the reviewers. Any product that may be evaluated in this article, or claim that may be made by its manufacturer, is not guaranteed or endorsed by the publisher.

Copyright (๑) 2022 Novo, Cavani, Pinedo, Melendez and Peñagaricano. This is an open-access article distributed under the terms of the Creative Commons Attribution License (CC BY). The use, distribution or reproduction in other forums is permitted, provided the original author(s) and the copyright owner(s) are credited and that the original publication in this journal is cited, in accordance with accepted academic practice. No use, distribution or reproduction is permitted which does not comply with these terms. 